\section{Prevalência de aleitamento materno e introdução precoce de suplementos alimentares em área urbana do Sudeste do Brasil, Embu, SP}

\section{Breast feeding prevalence and early supplementary feeding in an urban area of Southeast Brazil, Embu, SP}

Glaura César Pedroso 1

Rosana Fiorini Puccini 2

Edina Mariko Koga da Silva 3

Nilza Nunes da Silva 4

Maria Cecília Goi Porto Alves 5

1-3 Departamento de Pediatria. Universidade Federal de São Paulo. Rua Botucatu, 598. Vila Clementino. São Paulo, SP, Brasil. CEP. 04.023-062. Tel: (55) (11) 5549.6124

${ }^{4}$ Departamento de Epidemiologia. Faculdade de Saúde Pública Universidade de São Paulo, SP, Brasil

5 Superintendência de Controle de Endemias (SUCEN). São Paulo, SP, Brasil

\begin{abstract}
Objectives: to estimate breast feeding rates in Embu, SP, using World Health Organization indicators and to determine the association of "no breastfeeding" and "early introduction of food supplements" with healthcare social and demographic factors.

Methods: analytical household survey, with twostage systematic cluster sampling, as part of the project "Morbidity and Healthcare Facilities Use: a Survey on Child's Health". Participants - 798 children under three years old, living in Embu in 1996.

Results : breast feeding - 95,7\% (IC95\%: 93,298,2) of under one year old infants; exclusive breast feeding - 10,3\% (IC95\%: 2,9-17,6); predominant breast feeding - 32,3\% (IC95\%: 23,5-41,2); median breast feeding time: six months. Factors associated to the early introduction of supplements (use of other food, except for water or tea, before 120 days following birth): living in slum areas- OR $=3,70$ (IC95\%; 2,016,81); newborn hospital discharge five days following birth. OR =6,12 (IC95\%: 1,64-22,80); mothers over thirty years of age - OR = 1,70 (IC95\%: 1,01-2,86).

Conclusions: groups eligible for priority breast feeding education were determined pointing towards the need of further studies in addition to actions in slum area populations oriented towards prenatal and perinatal care improvement.
\end{abstract}

Key words Breast feeding, Nutrition surveys, Social indicators, Socioeconomic factors

\section{Resumo}

Objetivos: estimar a prevalência do aleitamento materno em Embu, utilizando indicadores da Organização Mundial de Saúde; investigar a associação dos eventos "não início do aleitamento materno" e "introdução precoce de suplementos alimentares" com fatores sociodemográficos e relacionados à assistência à saúde.

Métodos: estudo transversal analítico, domiciliar, com amostragem por conglomerados em dois estágios (sorteio sistemático) - parte do projeto "Morbidade e Utilização de Serviços de Saúde". População: 798 menores de três anos, residentes no Embu em 1996.

Resultados: aleitamento materno - 95,7\% (IC95\%: 93,2-98,2) dos menores de um ano; aleitamento exclusivo - 10,3\% (IC95\%: 2,9-17,6); predominante - 32,3\% (IC95\%: 23,5-41,2); duração mediana do aleitamento: seis meses. Fatores associados à introdução precoce de suplementos (uso de outros alimentos, exceto água ou chás, antes de 120 dias): residir em favela - OR = 3,70 (IC95\%; 2,01-6,81); alta hospitalar do recém-nascido após cinco dias de vida OR = 6,12 (IC95\%: 1,64-22,80); mãe com 30 anos ou mais - OR = 1,70 (IC95\%: 1,01-2,86).

Conclusões: foram caracterizados grupos prioritários para intervenção em aleitamento materno, apontando para a necessidade de novos estudos, além de ações dirigidas à população favelada e à melhora da assistência pré e perinatal.

Palavras-chave Aleitamento materno, Inquéritos nutricionais, Indicadores sociais, Fatores socioeconômicos 


\section{Introdução}

Nas últimas décadas, a superioridade do aleitamento natural vem sendo demonstrada em vários aspectos: nutrição adequada, proteção contra infecções, modulação da resposta imune, fortalecimento do vínculo mãe-filho, contribuições para o desenvolvimento da criança e outros. ${ }^{1,2}$ A melhora das taxas de aleitamento materno também contribui para a redução da mortalidade infantil, para o aumento do intervalo interpartal e tem considerável impacto ambiental. ${ }^{2}$ Escuder et al. ${ }^{3}$ afirmam que a amamentação no primeiro ano de vida pode ser a estratégia mais exeqüível de redução da mortalidade pós-neonatal para além dos níveis já alcançados em municípios do Estado de São Paulo.

As transformações demográficas ocorridas na periferia dos centros urbanos desde a década de 70 têm influência sobre o perfil epidemiológico dessas regiões, além de contribuir para o agravamento da tensão social e familiar e para a perda de tradições, vínculos, crenças e valores, com redução dos mecanismos de apoio social à mãe que amamenta.2,4 Esse processo foi parcialmente revertido em anos mais recentes por meio de atividades de promoção, proteção e apoio ao aleitamento, com participação do governo brasileiro 5 e de movimentos liderados por mães e por profissionais da saúde. Assim, a duração mediana do aleitamento materno aumentou para dez meses nas capitais brasileiras, embora o aumento da prevalência da amamentação exclusiva ainda represente um desafio. 6

A importância de informações populacionais seguras sobre a situação do aleitamento materno, aliada ao conhecimento das dificuldades associadas a essa prática, reside em possibilitar o direcionamento e avaliação das ações de incentivo, visando a melhoria da situação de saúde com maior eqüidade, por meio de ações que levem em conta as diferenças e riscos individuais ou populacionais.

A identificação de fatores de risco individuais já é amplamente utilizada nos serviços de saúde com a finalidade de abordar os indivíduos e grupos, segundo sua maior ou menor probabilidade de adoecer. Com menor freqüência são utilizados os indicadores de risco coletivo, que, em geral, apontam para necessidades que exigem políticas intersetoriais dirigidas a áreas de risco que atendem os contrastes de condições de vida e sua influência sobre a situação de saúde.

Um inquérito realizado no município de Embu, em 1995, durante campanha nacional de vacinação, indicou que $46 \%$ das crianças menores de quatro meses estavam em aleitamento materno, sem o uso de leite heterólogo, não havendo informações sobre os alimentos não lácteos. A duração mediana do aleitamento materno foi calculada em seis meses. $\mathrm{O}$ número de crianças sem leite materno aos seis meses de idade era de $20 \% .7$

Este estudo teve como objetivos: descrever a situação do aleitamento materno no município de Embu, utilizando os indicadores propostos pela World Health Organization (WHO), 8 investigar a associação dos eventos "não início do aleitamento materno" e "introdução precoce de suplementos alimentares" com fatores sócio-demográficos e relacionados à assistência à saúde.

\section{Métodos}

O presente estudo foi aprovado pelo Comitê de Ética em Pesquisa da Universidade Federal de São Paulo (UNIFESP) e é parte integrante do projeto "Morbidade e Utilização de Serviços no Município de Embu: Estudo Epidemiológico na área de Saúde Infantil", 9 que envolveu pesquisadores e profissionais da Faculdade de Saúde Pública da Universidade de São Paulo, da UNIFESP e da Prefeitura Municipal da Estância Turística de Embu, com financiamento da FAPESP (Fundação de Amparo à Pesquisa do Estado de São Paulo).

\section{O município}

Embu é um município da Região Sudoeste da Região Metropolitana de São Paulo, SP, Brasil, com população de 194.879 habitantes em 1996. Nesse mesmo ano, o coeficiente de mortalidade infantil foi de 28,12 por 1.000 nascidos vivos e vem diminuindo progressivamente, atingindo 13,98 por mil nascidos vivos em 2002. Dois terços desse número correspondem à mortalidade neonatal. ${ }^{10}$ As principais causas de óbito no período pós-neonatal, no ano de 1996, foram as pneumonias e outras afecções respiratórias e, na faixa etária de um a quatro anos, pneumonia e acidentes em geral. A mortalidade por homicídios em adolescentes e adultos jovens é bastante elevada, mostrando a situação de violência e exclusão social em que vive a maior parte da população.

A rede de assistência à saúde do Município era composta, no ano do estudo, por nove unidades básicas de saúde e dois prontos-socorros, sendo a assistência hospitalar prestada pelos municípios vizinhos. Não havia hospitais participantes da "Iniciativa Hospital Amigo da Criança" na Região. Desde 1970, a UNIFESP mantém no Município um programa de integração docente-assistencial envolvendo vários de- 
partamentos e disciplinas.

A participação da comunidade junto ao sistema de saúde ainda é limitada, embora o Município possua um Conselho de Saúde atuante, com participação efetiva da sociedade civil.

\section{Estudo do Município por estratos} de condições de vida

Dispondo de arquivos de dados do censo demográfico de 1991, a população foi estratificada em agrupamentos homogêneos definidos segundo condições de moradia, escolaridade e situação socioeconômica da família. As variáveis utilizadas foram: tipo de moradia, instalações sanitárias, destino do lixo, sanitário dentro do domicílio, rede geral de abastecimento de água, destino do esgoto, escolaridade do chefe da família (em anos) e renda familiar em salários mínimos. 9 Com isto, os 135 setores censitários que compõem a área geográfica do Município foram reunidos através da técnica de análise de agrupamentos, adotando-se o método hierárquico Average linkage. A aplicação desse processo para a população estudada é detalhada em trabalho de Frei.11

Desse modo, os 135 setores censitários foram agrupados em quatro estratos, que possuíam os seguintes perfis: 9

a) Estrato um (17 setores): menor densidade demográfica; renda familiar mais elevada e melhores condições ambientais; uma criança menor de cinco anos para cada três domicílios; maior número de chefes de família com quinze anos ou mais de escolaridade;

b) Estratos dois (67 setores) e três (34 setores): uma criança menor de cinco anos para cada dois domicílios; renda familiar entre quatro e sete salários mínimos; predominavam chefes de família com até o primeiro grau de escolaridade (atualmente chamado ensino fundamental); mais heterogêneos, refletindo as condições ambientais predominantes no Município. Apesar de sua semelhança, esses estratos foram mantidos separados devido à presença de favelas no estrato três.

c) Estrato 4 (17 setores): 1,3 menores de cinco anos para cada dois domicílios; todas as moradias foram classificadas como aglomerados subnormais (favelas) no censo demográfico de 1991; setenta e nove por cento dos chefes de família possuía até três anos de escolaridade e $23,2 \%$, renda de até um salário mínimo; as condições sociais e ambientais eram as piores do Município.

\section{Processo de amostragem e estimação dos pesos}

Devido a especificidades da morbi-mortalidade e do padrão de utilização de serviços de saúde, originalmente foram consideradas duas populações independentes: crianças menores de um ano e crianças de um a quatro anos, residentes no município de Embu em 1996. Mantendo-se em 5\% o limite tolerado para os erros dos resultados amostrais obtidos em cada grupo, fixou-se em $10 \%$ o erro aceitável para as estimativas calculadas para cada estrato; se essas estimativas são de proporções populacionais, estimouse que o número de crianças observadas deveria ser, no mínimo, igual a 81 crianças para cada estrato e 323 para o total de cada grupo etário, caso essas proporções (ou seus complementares) não ultrapassem $30 \%$.

O processo de sorteio adotado no estudo original foi o de conglomerados em dois estágios. No primeiro, dez setores censitários foram sorteados, em cada estrato, sob o critério de partilha proporcional aos seus tamanhos. No segundo estágio, foram sorteados domicílios de cada setor sorteado.

A amostragem por conglomerados em dois estágios, com atualização em campo do tamanho dos setores censitários e com sorteio de igual número de domicílios em cada estrato, introduziu diferenças de probabilidade $(f)$ de qualquer família ou criança da população pertencer à amostra sorteada. Para cada estrato, o peso $w$ foi calculado pelo inverso da probabilidade $f$. Ou seja, $w=1 / f$. A expressão matemática adotada para calcular $f$ decorreu do plano de amostragem adotado. 9

Para avaliar o aleitamento materno, consideramos apenas as crianças com idade até 35 meses e 29 dias, levando-se em conta os possíveis erros pela utilização de dados recordatórios e a metodologia estabelecida pela WHO para construção de indicadores de aleitamento materno. ${ }^{8}$

Foram excluídos da amostra os filhos adotivos, crianças internadas na época da entrevista e questionários sem informações sobre o aleitamento materno por não ter sido possível entrevistar a mãe. Esses casos corresponderam a 3,3\% das 825 crianças menores de 36 meses, restando uma amostra final de 798 crianças.

\section{Coleta e análise de dados}

As questões referentes ao aleitamento materno foram respondidas, obrigatoriamente, pelas mães. O questionário incluía dados sobre a alimentação atual da criança e também sobre o histórico de amamentação e desmame, conforme lembrado pelas mães no mo- 
mento da entrevista, que era realizada no domicílio. Como o questionário do projeto inicial não era o recomendado pela $\mathrm{WHO}^{8}$ (que se baseia nos alimentos recebidos nas últimas 24 horas), foi feita, após a coleta dos dados, uma adaptação das respostas para refletir o status corrente do aleitamento. Variáveis com perda de mais de $20 \%$ das respostas não foram consideradas na análise.

Foram calculados os seguintes indicadores de aleitamento materno: 8 taxa de aleitamento materno exclusivo - proporção dos lactentes menores de 120 dias de idade, amamentados exclusivamente nas últimas 24 horas; b) taxa de aleitamento materno predominante - proporção dos lactentes menores 120 dias, amamentados de modo predominante (isto é, leite materno mais outros fluidos, como água, chás, terapia de reidratação oral); c) taxa de aleitamento materno exclusivo e predominante (soma das taxas de aleitamento exclusivo e predominante); d) taxa de alimentação complementar oportuna - proporção dos lactentes de seis a nove meses recebendo alimentos complementares em adição ao leite materno; e) taxa de aleitamento materno continuado (um ano) - proporção das crianças de 12 a 15 meses que estão sendo amamentadas; f) taxa de aleitamento materno continuado (dois anos) - proporção das crianças de 20 a 23 meses que estão sendo amamentadas; g) taxa de aleitamento ever breast-fed rate - proporção das crianças menores de 12 meses que foram, em algum momento, amamentadas; h) Taxa de primeira mamada oportuna - proporção das crianças menores de 12 meses amamentadas na primeira hora após o nascimento; i) duração mediana do aleitamento materno idade, em meses, em que $50 \%$ das crianças, ou mais, já não são amamentadas (é calculada utilizando-se a média móvel de três meses para a prevalência de aleitamento materno).

Para avaliação da introdução precoce de suplementos alimentares, avaliamos as crianças entre quatro meses e 35 meses e 29 dias (627 crianças). Consideramos precoce, neste estudo, a introdução de alimentos, exceto água ou chás, em adição ou substituição ao leite materno, antes de 120 dias de vida. O oferecimento de água e chás, embora não recomendado, é bastante difundido na população estudada e por isso não foi considerado na parte analítica deste trabalho.

A entrada e análise dos dados do projeto original e da parte descritiva foram processados utilizando-se o programa Epi-info 6, versão 6.04, incluindo o módulo CSAMPLE. A análise dos fatores associados ao não início do aleitamento materno e à introdução precoce de suplementos alimentares foi feita utilizando-se o mesmo programa. A significância es- tatística foi verificada por meio dos intervalos de confiança.

Foi realizada análise multivariada, por meio de regressão logística, utilizando o software WesVarPC, que permite levar em conta o delineamento complexo do estudo original. Para inclusão das variáveis independentes no modelo de regressão logística, foi adotado o nível de significância de $10 \%(\mathrm{p} \leq 0,10)$. As variáveis anteriormente utilizadas na construção dos estratos não foram consideradas na análise multivariada.

\section{Resultados}

A amostra final estava constituída por $51,4 \%$ de crianças do sexo masculino e $48,6 \%$ do sexo feminino. Os menores de um ano foram representados por 468 crianças; as crianças de um ano até dois anos incompletos, por 162 crianças; as crianças de dois anos, por 168 crianças. A distribuição por estratos de condições de vida foi: Estrato 1 - 147 famílias e 159 crianças; Estrato 2 - 182 famílias e 194 crianças; Estrato 3 - 184 famílias e 193 crianças; Estrato 4 - 232 famílias e 252 crianças.

As principais características da população estudada são exibidas nas Tabelas 1 e 2 . A prevalência do aleitamento materno, descrita por meio dos indicadores da WHO, está na Tabela 3, na qual podemos observar também que 95,2\% (IC95\%: 92,4-98,0) das crianças menores de três anos iniciaram o aleitamento materno após o nascimento, mas 69,4\% (IC95\%: $65,4-73,4)$ receberam outros alimentos, além de água ou chás, antes de 120 dias de vida.

Não houve associação estatisticamente significante entre os fatores estudados e o não início do aleitamento materno, como mostram as Tabelas 4 e 5.

Os fatores associados à introdução precoce de suplementos alimentares são exibidos nas Tabelas 6 e 7.

Após análise multivariada pelo método de regressão logística, observamos, como mostra a Tabela 8 , que residir em favela foi o principal fator associado à introdução precoce de suplementos alimentares.

Também apresentaram associação estatisticamente significante as variáveis: permanência em berçário por mais de cinco dias e mãe com 30 anos ou mais. 


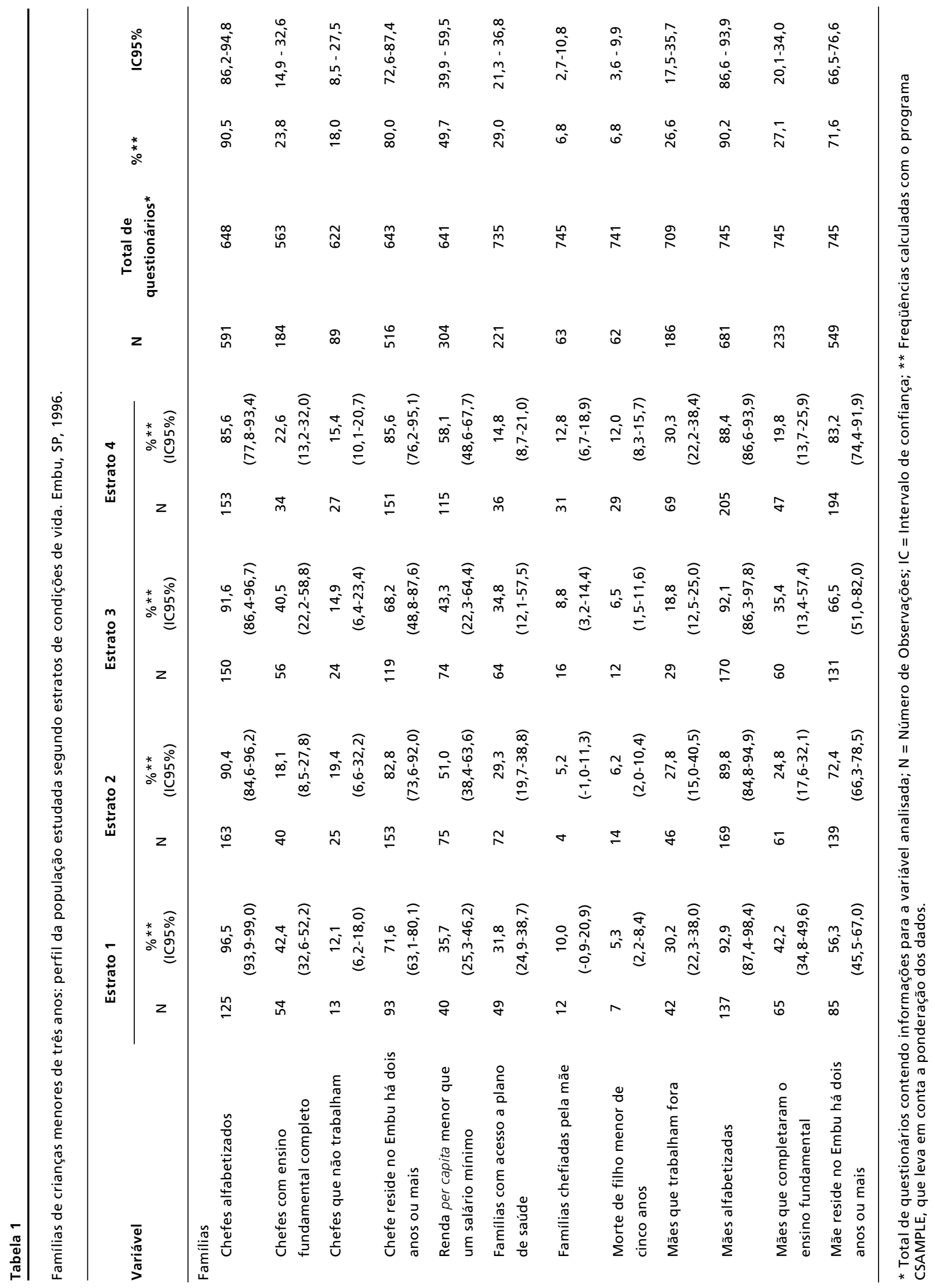




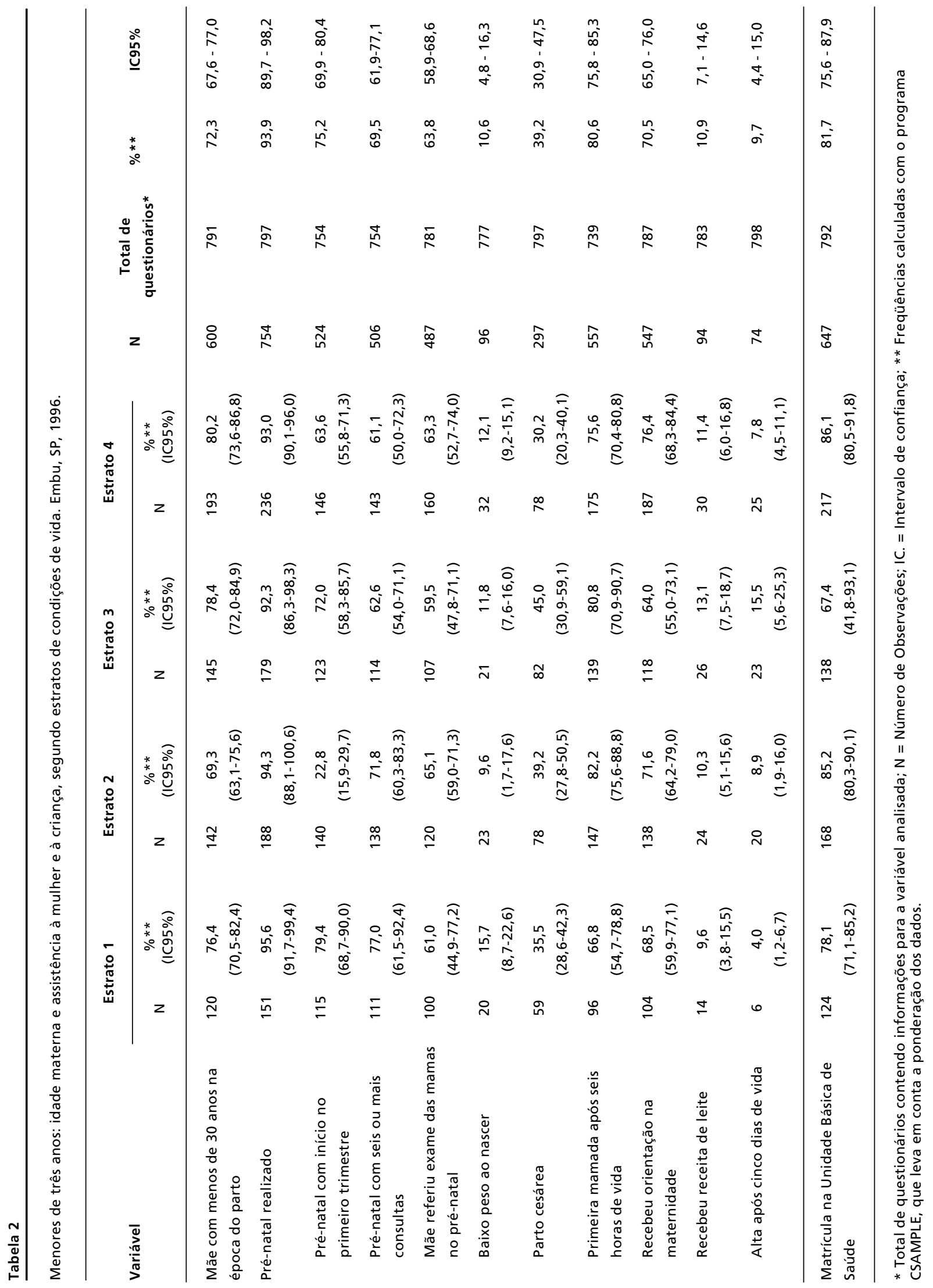


Prevalência de aleitamento materno, início do aleitamento materno e introdução precoce de suplementos. Embu, SP, 1996.

\begin{tabular}{|c|c|c|}
\hline Indicador & Prevalência (\%) & IC95\% \\
\hline Taxa de aleitamento exclusivo & 10,3 & $2,9-17,6$ \\
\hline Taxa de aleitamento predominante & 32,3 & $23,5-41,2$ \\
\hline Taxa de aleitamento exclusivo e predominante & 42,6 & $30,3-54,9$ \\
\hline Alimentação complementar oportuna & 35,0 & $19,6-50,6$ \\
\hline Aleitamento continuado (um ano) & 27,6 & $1,9-53,3$ \\
\hline Aleitamento continuado (dois anos) & 4,1 & $-1,0-9,2$ \\
\hline Taxa de aleitamento Ever breast-fed rate & 95,7 & $93,2-98,2$ \\
\hline Primeira mamada oportuna & 3,6 & $1,7-5,4$ \\
\hline Duração mediana do aleitamento materno* & 6 meses & \\
\hline
\end{tabular}

Freqüências para o total da amostra **

Iniciaram o aleitamento

95,2

$92,4-98,0$

Introdução precoce de suplementos

69,4

$65,4-73,4$

* Idade, em meses, em que $50 \%$ ou mais das crianças não são amamentadas; ** Para avaliar a introdução precoce de suplementos, foram consideradas apenas as crianças maiores de 120 dias (627 crianças).

\section{Discussão}

\section{A situação do aleitamento materno no Município de Embu}

Os indicadores da WHO representam um avanço importante nas pesquisas sobre aleitamento materno por eliminar o viés dos dados recordatórios, uniformizar os termos utilizados na literatura, permitir a comparação entre trabalhos e o estabelecimento de metas para os esforços de promoção.

A prevalência do aleitamento exclusivo no Brasil é uma das mais baixas da América Latina ${ }^{12}$ devido, provavelmente, ao processo de urbanização e seu efeito sobre a composição familiar, a cultura e as tradições locais. Os resultados obtidos no Embu refletem esse padrão, com uma prevalência de aleitamento materno exclusivo muito baixa (10,3\% entre menores de quatro meses). Mesmo se somarmos as crianças que recebem água ou chás, obteremos uma taxa de aleitamento exclusivo e predominante de 42,6\% (IC95\%: 30,3-54,9). Esses valores estão abaixo da estimativa nacional,13 devendo-se salientar as importantes diferenças entre cidades quanto à prevalência e duração do aleitamento. 14
Um estudo realizado em 84 municípios do estado de São Paulo, em 1998, evidenciou taxas de aleitamento exclusivo variando de $0 \%$ a $54 \%$ (sendo maior que $20 \%$ em 27 municípios), com grande heterogeneidade mesmo dentro de uma mesma região. A taxa de aleitamento predominante foi maior que $20 \%$ na maioria $(85,7 \%)$ dos municípios estudados. 15

Isso indica que, embora estejamos vivendo um período de retomada da amamentação, ainda há muito por fazer para melhorar os indicadores de aleitamento materno em nossa Região, merecendo destaque as políticas locais de incentivo ao aleitamento e de promoção da saúde.

\section{Fatores sócio-demográficos e assistenciais associados ao aleitamento}

Diversas condições já foram descritas como associadas à maior probabilidade de não iniciar o aleitamento materno ou de interrompê-lo precocemente. ${ }^{15,16} \mathrm{~A}$ literatura disponível é bastante heterogênea e a comparabilidade entre os resultados dos diferentes autores é limitada, uma vez que o desmame é um processo social17 e os estudos utilizam métodos 
Distribuição de crianças menores de 36 meses quanto ao início do aleitamento materno. Embu, SP, 1996.

\begin{tabular}{|c|c|c|c|c|c|c|}
\hline \multirow{2}{*}{ Indicador } & \multicolumn{2}{|c|}{ Iniciou } & \multicolumn{2}{|c|}{ Não Iniciou } & \multirow{2}{*}{ OR } & \multirow{2}{*}{ IC95\% } \\
\hline & $\mathrm{N}$ & $(\%) *$ & $\mathrm{~N}$ & $(\%) *$ & & \\
\hline \multicolumn{7}{|l|}{ Estrato de condiçõos de vida } \\
\hline Estrato 4 (favela) & 235 & 92,6 & 17 & 7,4 & 0,55 & $0,20-1,53$ \\
\hline Outros estratos & 514 & 95,7 & 32 & 4,3 & & \\
\hline \multicolumn{7}{|l|}{ Renda per capita } \\
\hline Menor que um salário mínimo & 306 & 93,2 & 26 & 6,8 & 0,42 & $0,14-1,30$ \\
\hline Maior ou igual a um salário mínimo & 342 & 97,0 & 15 & 3,0 & & \\
\hline \multicolumn{7}{|l|}{ Alfabetização materna } \\
\hline Mãe alfabetizada & 681 & 95,2 & 44 & 4,7 & 0,62 & $0,19-2,11$ \\
\hline Mãe não alfabetizada & 68 & 97,0 & 5 & 3,0 & & \\
\hline \multicolumn{7}{|l|}{ Alfabetização do(a) chefe da família } \\
\hline Chefe alfabetizado(a) & 598 & 95,6 & 32 & 4,4 & 1,38 & $0,40-4,72$ \\
\hline Chefe não alfabetizado(a) & 58 & 94,0 & 6 & 4,0 & & \\
\hline \multicolumn{7}{|l|}{ Escolaridade materna } \\
\hline Mãe com quarta série completa & 585 & 96,2 & 34 & 3,8 & 2,01 & $0,72-5,63$ \\
\hline Mãe com até terceira série completa & 164 & 92,7 & 15 & 7,3 & & \\
\hline \multicolumn{7}{|l|}{ Escolaridade do(a) chefe da família } \\
\hline Chefe com a quarta série completa & 453 & 95,0 & 24 & 5,0 & 1,01 & $0,37-2,77$ \\
\hline Chefe com até terceira série completa & 116 & 94,9 & 8 & 5,1 & & \\
\hline \multicolumn{7}{|l|}{ Posição da mãe como chefe de família } \\
\hline Mãe chefe da família & 63 & 98,1 & 3 & 1,9 & 2,61 & $0,71-9,66$ \\
\hline Mãe não é chefe da família & 686 & 95,2 & 46 & 4,8 & & \\
\hline \multicolumn{7}{|l|}{ Idade materna } \\
\hline 30 anos ou mais à época do parto & 169 & 92,5 & 22 & 7,5 & 0,45 & $0,15-1,38$ \\
\hline Menos de 30 anos à época do parto & 574 & 96,4 & 26 & 3,5 & & \\
\hline \multicolumn{7}{|l|}{ Óbito de filho menor de cinco anos } \\
\hline Sim & 61 & 94,5 & 7 & 5,5 & 0,80 & $0,25-2,66$ \\
\hline Não & 688 & 95,5 & 42 & 4,5 & & \\
\hline Total & 749 & 95,2 & 49 & 4,8 & & \\
\hline
\end{tabular}

$\mathrm{N}$ = Número de observações; OR = Odds ratio; IC = Intervalo de confiança; * Freqüências calculadas com o programa CSAMPLE, que leva em conta a ponderação dos dados.

diferentes e se referem a populações que vivem contextos históricos, sociais e culturais diversos.

Carvalho et al. 4 agrupam os fatores determinantes da interrupção do aleitamento em dois grupos: fatores que influem na decisão pessoal da mãe e que estão relacionados com o nível individual, familiar ou comunitário; e fatores socioeconômicos que afetam indiretamente a decisão da mãe, contribuindo para a perda da cultura e da tradição do aleitamento materno. Procuramos aqui contribuir para o estudo de al- 
Início do aleitamento materno, segundo variáveis assistenciais e ordem de nascimento da criança, menores de 36 meses. Embu, SP, 1996.

\begin{tabular}{|c|c|c|c|c|c|c|}
\hline \multirow{2}{*}{ Indicador } & \multicolumn{2}{|c|}{ Iniciou } & \multicolumn{2}{|c|}{ Não Iniciou } & \multirow{2}{*}{ OR } & \multirow{2}{*}{ IC95\% } \\
\hline & $\mathrm{N}$ & $(\%) *$ & $\mathrm{~N}$ & $(\%) *$ & & \\
\hline \multicolumn{7}{|l|}{ Pré-natal } \\
\hline Pré-natal realizado & 707 & 95,2 & 47 & 4,7 & 0,22 & $0,03-1,81$ \\
\hline Pré-natal não realizado & 42 & 98,9 & 1 & 1,1 & & \\
\hline \multicolumn{7}{|l|}{ Exame das mamas durante o pré-natal } \\
\hline Mamas examinadas no pré-natal & 459 & 95,8 & 28 & 4,2 & 1,22 & $0,54-2,78$ \\
\hline Mamas não examinadas no pré-natal & 275 & 94,9 & 19 & 5,1 & & \\
\hline \multicolumn{7}{|l|}{ Época de início do pré-natal } \\
\hline Primeiro trimestre & 493 & 95,4 & 31 & 4,6 & 1,16 & $0,41-3,25$ \\
\hline Após o primeiro trimestre & 214 & 94,7 & 16 & 5,3 & & \\
\hline \multicolumn{7}{|l|}{ Número de consultas de pré-natal } \\
\hline Seis ou mais consultas & 477 & 95,6 & 29 & 4,3 & 1,37 & $0,53-3,58$ \\
\hline Até cinco consultas & 230 & 93,3 & 18 & 6,7 & & \\
\hline \multicolumn{7}{|l|}{ Ordem de nascimento } \\
\hline Primeiro filho & 290 & 94,3 & 20 & 5,7 & 0,76 & $0,27-2,18$ \\
\hline Outros filhos & 459 & 95,6 & 29 & 4,4 & & \\
\hline \multicolumn{7}{|l|}{ Baixo peso ao nascer } \\
\hline Sim & 85 & 93,7 & 11 & 6,2 & 0,69 & $0,22-2,24$ \\
\hline Não & 645 & 95,6 & 36 & 4,4 & & \\
\hline \multicolumn{7}{|l|}{ Tipo de parto } \\
\hline Parto cesárea & 271 & 93,9 & 26 & 6,1 & 0,57 & $0,18-1,79$ \\
\hline Parto vaginal & 478 & 96,4 & 22 & 3,6 & & \\
\hline \multicolumn{7}{|l|}{ Primeira mamada } \\
\hline Após seis horas de vida & 534 & 97,4 & 23 & 2,6 & 2,50 & $0,63-9,98$ \\
\hline Até seis horas de vida & 174 & 93,7 & 8 & 6,2 & & \\
\hline \multicolumn{7}{|l|}{ Orientação para aleitamento } \\
\hline Recebeu orientação no hospital & 516 & 96,1 & 31 & 3,8 & 1,81 & $0,81-4,07$ \\
\hline Não recebeu orientação no hospital & 224 & 93,2 & 16 & 6,7 & & \\
\hline \multicolumn{7}{|l|}{ Prescrição de leite heterólogo } \\
\hline Recebeu receita de leite & 83 & 93,0 & 11 & 7,0 & 0,62 & $0,21-1,85$ \\
\hline Não recebeu receita de leite & 653 & 95,5 & 36 & 4,5 & & \\
\hline \multicolumn{7}{|l|}{ Alta hospitalar do recém-nascido } \\
\hline Após cinco dias de vida & 62 & 90,3 & 12 & 9,7 & 0,41 & $0,15-1,16$ \\
\hline Até cinco dias de vida & 687 & 95,8 & 37 & 4,2 & & \\
\hline Total & 749 & 95,2 & 49 & 4,8 & & \\
\hline
\end{tabular}

N = Número de observações; OR = Odds ratio; IC = Intervalo de confiança; * Freqüências calculadas com o programa CSAMPLE, que leva em conta a ponderação dos dados.

guns desses fatores, numa tentativa de direcionar os esforços para melhorar as taxas de aleitamento materno.

Nenhuma das variáveis estudadas apresentou associação estatisticamente significante com o não iní- cio do aleitamento materno. Esse resultado se deve, provavelmente, ao tamanho amostral, já que o número de crianças que não iniciou o aleitamento materno é muito pequeno.

Venancio et al.,15 avaliando as prevalências de 
Introdução de suplementos antes de 120 dias de vida, exceto água e chás, segundo variáveis sociodemográficas. Crianças entre 4 e 35 meses completos. Embu, SP, 1996.

\begin{tabular}{|c|c|c|c|c|c|c|}
\hline \multirow{2}{*}{ Variável } & \multicolumn{2}{|c|}{ Precoce } & \multicolumn{2}{|c|}{ Não precoce } & \multirow{2}{*}{ OR } & \multirow{2}{*}{ IC95\% } \\
\hline & $\mathrm{N}$ & $(\%)^{*}$ & $\mathrm{~N}$ & $(\%)^{*}$ & & \\
\hline \multicolumn{7}{|l|}{ Estrato de condições de vida } \\
\hline Estrato 4 (favela) & 170 & 84,5 & 34 & 15,5 & 2,26 & $1,32-3,89$ \\
\hline Outros estratos & 288 & 70,7 & 135 & 29,3 & & \\
\hline \multicolumn{7}{|l|}{ Renda per capita } \\
\hline Menor que um salário mínimo & 196 & 76,3 & 63 & 23,7 & 1,61 & $0,63-4,14$ \\
\hline Maior ou igual a um salário mínimo & 207 & 66,6 & 84 & 33,4 & & \\
\hline \multicolumn{7}{|l|}{ Alfabetização materna } \\
\hline Mãe alfabetizada & 414 & 71,1 & 151 & 28,9 & 0,58 & $0,20-1,76$ \\
\hline Mãe não alfabetizada & 44 & 80,7 & 18 & 19,3 & & \\
\hline \multicolumn{7}{|l|}{ Alfabetização do(a) chefe da família } \\
\hline Chefe alfabetizado(a) & 347 & 70,8 & 144 & 29,2 & 0,71 & $0,37-1,38$ \\
\hline Chefe não alfabetizado(a) & 46 & 77,2 & 8 & 22,8 & & \\
\hline \multicolumn{7}{|l|}{ Escolaridade da mãe } \\
\hline Mãe com quarta série completa & 350 & 72,4 & 129 & 27,6 & 1,07 & $0,60-1,91$ \\
\hline Mãe com até terceira série completa & 108 & 71,0 & 40 & 29,0 & & \\
\hline \multicolumn{7}{|l|}{ Escolaridade do(a) chefe da família } \\
\hline Chefe com quarta série completa & 262 & 71,5 & 113 & 28,5 & 1,09 & $0,32-3,75$ \\
\hline Chefe com até terceira série completa & 68 & 69,7 & 25 & 30,3 & & \\
\hline \multicolumn{7}{|l|}{ Posição da mãe como chefe da família } \\
\hline Mãe chefe da família & 42 & 74,5 & 12 & 25,5 & 1,14 & $0,57-2,31$ \\
\hline Mãe não é chefe da família & 416 & 71,9 & 157 & 28,1 & & \\
\hline \multicolumn{7}{|l|}{ Idade materna } \\
\hline 30 anos ou mais à época do parto & 109 & 76,1 & 39 & 23,8 & 1,57 & $1,02-2,43$ \\
\hline Menos de 30 anos à época do parto & 347 & 67,0 & 33 & 33,0 & & \\
\hline \multicolumn{7}{|l|}{ Óbito de filho menor de cinco anos } \\
\hline Sim & 37 & 64,4 & 13 & 35,6 & 0,68 & $0,21-2,27$ \\
\hline Não & 421 & 69,2 & 156 & 30,8 & & \\
\hline Total & 458 & 69,4 & 169 & 30,6 & & \\
\hline
\end{tabular}

$\mathrm{N}=$ Número de observações; OR = Odds ratio; IC = Intervalo de confiança; * Freqüências calculadas com o programa CSAMPLE, que leva em conta a ponderação dos dados.

aleitamento exclusivo em 84 municípios paulistas, encontraram, como fatores associados à interrupção precoce do aleitamento exclusivo: menor escolaridade materna, residir em municípios que não possuem Hospital Amigo da Criança, nascer em outro tipo de hospital, primiparidade e idade materna inferior a vinte anos. Esses resultados diferem dos encontrados no Embu, embora os trabalhos não sejam rigorosamente comparáveis.
Observou-se maior freqüência de introdução precoce de suplementos alimentares entre os moradores de favelas, que apresentam as piores condições de vida do Município. Essas condições podem interferir negativamente no aleitamento de vários modos: pior acesso à informação, deficiências na atenção à saúde, 9,18 desestruturação familiar, baixa auto-estima, suporte social precário e dificuldades em conciliar as tarefas relacionadas à sobre- 
Introdução de suplementos antes de 120 dias de vida, exceto água e chás, segundo variáveis assistenciais e ordem de nascimento da criança. Crianças entre 4 e 35 meses completos. Embu, SP, 1996.

\begin{tabular}{|c|c|c|c|c|c|c|}
\hline \multirow{2}{*}{ Variável } & \multicolumn{2}{|c|}{ Precoce } & \multicolumn{2}{|c|}{ Não precoce } & \multirow{2}{*}{ OR } & \multirow{2}{*}{ IC95\% } \\
\hline & $\mathrm{N}$ & $(\%) *$ & $\mathrm{~N}$ & $(\%) *$ & & \\
\hline \multicolumn{7}{|l|}{ Pré-natal } \\
\hline Pré-natal realizado & 428 & 67,9 & 163 & 32,1 & 0,25 & $0,03-1,91$ \\
\hline Pré-natal não realizado & 29 & 89,2 & 6 & 10,8 & & \\
\hline \multicolumn{7}{|l|}{ Exame das mamas durante o pré-natal } \\
\hline Mamas examinadas no pré-natal & 272 & 62,3 & 113 & 37,7 & 0,37 & $0,15-0,90$ \\
\hline Mamas não examinadas no pré-natal & 175 & 81,8 & 53 & 18,3 & & \\
\hline \multicolumn{7}{|l|}{ Época de início do pré-natal } \\
\hline Primeiro trimestre & 308 & 68,6 & 117 & 31,3 & 1,16 & $0,62-2,19$ \\
\hline Após o primeiro trimestre & 120 & 65,3 & 46 & 34,7 & & \\
\hline \multicolumn{7}{|l|}{ Número de consultas de pré-natal } \\
\hline Seis ou mais consultas & 288 & 71,4 & 117 & 28,6 & 1,71 & $0,54-5,45$ \\
\hline Até cinco consultas & 140 & 59,2 & 46 & 40,8 & & \\
\hline \multicolumn{7}{|l|}{ Ordem de nascimento } \\
\hline Primeiro filho & 176 & 64,8 & 69 & 35,2 & 0,73 & $0,38-1,41$ \\
\hline Outros filhos & 282 & 71,5 & 100 & 28,5 & & \\
\hline \multicolumn{7}{|l|}{ Baixo peso ao nascer } \\
\hline Sim & 64 & 75,2 & 18 & 24,8 & 1,40 & $0,52-3,79$ \\
\hline Não & 379 & 68,3 & 147 & 31,6 & & \\
\hline \multicolumn{7}{|l|}{ Tipo de parto } \\
\hline Parto cesárea & 178 & 69,5 & 58 & 30,5 & 1,01 & $0,52-1,96$ \\
\hline Parto vaginal & 279 & 69,2 & 111 & 30,8 & & \\
\hline \multicolumn{7}{|l|}{ Primeira mamada } \\
\hline Após seis horas de vida & 325 & 68,6 & 116 & 31,4 & 1,19 & $0,35-4,09$ \\
\hline Até seis horas de vida & 90 & 64,6 & 45 & 35,4 & & \\
\hline \multicolumn{7}{|l|}{ Orientação para aleitamento } \\
\hline Recebeu orientação no hospital & 328 & 70,6 & 112 & 29,4 & 1,24 & $0,76-2,02$ \\
\hline Não recebeu orientação no hospital & 123 & 65,9 & 56 & 34,1 & & \\
\hline \multicolumn{7}{|l|}{ Prescrição de leite heterólogo } \\
\hline Recebeu receita de leite & 67 & 77,8 & 13 & 22,2 & 1,62 & $0,32-8,24$ \\
\hline Não recebeu receita de leite & 380 & 68,4 & 154 & 31,6 & & \\
\hline \multicolumn{7}{|l|}{ Alta hospitalar } \\
\hline Após cinco dias de vida & 54 & 92,1 & 7 & 7,9 & 5,84 & $1,81-18,9$ \\
\hline Até cinco dias de vida & 404 & 66,7 & 162 & 33,3 & & \\
\hline Total & 458 & 69,4 & 169 & 30,6 & & \\
\hline
\end{tabular}

$\mathrm{N}$ = Número de observações; OR = Odds ratio; IC = Intervalo de confiança * Freqüências calculadas com o programa CSAMPLE, que leva em conta a ponderação dos dados. 
Resultados da análise de regressão logística para introdução precoce de suplementos em crianças maiores de quatro meses de vida. Embu, SP, 1996.

\begin{tabular}{|c|c|c|c|c|c|c|}
\hline \multirow{2}{*}{ Variável } & \multicolumn{2}{|c|}{ Precoce } & \multirow{2}{*}{ Coeficiente } & \multirow{2}{*}{ Valor de $p$} & \multirow{2}{*}{ OR } & \multirow{2}{*}{ IC95\% } \\
\hline & $\begin{array}{l}\text { Sim } \\
\text { (n) }\end{array}$ & $\begin{array}{c}\text { Não } \\
\text { (n) }\end{array}$ & & & & \\
\hline Residir em favela & 170 & 34 & $-0,415$ & 0,000 & 3,70 & $2,01-6,81$ \\
\hline Alta hospitalar após cinco dias de vida & 54 & 7 & $-0,881$ & 0,008 & 6,12 & $1,64-22,80$ \\
\hline Mãe com 30 anos ou mais & 109 & 39 & $-0,397$ & 0,045 & 1,70 & $1,01-2,86$ \\
\hline
\end{tabular}

$\mathrm{n}$ = número de observações; $O R=$ Odds ratio; IC = Intervalo de confiança.

vivência familiar com a amamentação - embora essa seja a melhor estratégia para a sobrevivência infantil.

Também não podemos descartar a possível participação de outros fatores, tais como diferenças no cuidado da criança ou no uso dos serviços de saúde que não tenham sido avaliadas no presente trabalho.

Essas informações evidenciam a necessidade de intervenções para promoção da saúde e inclusão social da população favelada, para a qual o papel protetor do aleitamento materno é muito importante.

A permanência em berçário por mais de cinco dias foi um fator associado à introdução precoce de suplementos. Tal resultado era esperado, pois as mesmas intercorrências neonatais que levam ao maior tempo de permanência na maternidade podem levar a dificuldades na lactação, por problemas clínicos ou separação entre mãe e filho. A permanência da criança no hospital após a alta materna agrava essa separação, principalmente se levarmos em conta as grandes distâncias entre a residência da família e o local do parto, a presença de outros filhos que necessitam de cuidado, a ausência de ajuda na execução dos afazeres domésticos e a ausência de políticas efetivas de promoção do aleitamento na maioria dos hospitais utilizados pela população do Embu, naquele período. Freqüentemente, os recém-nascidos que permaneciam internados recebiam alta em aleitamento misto ou artificial, não havendo, após a alta, apoio dos serviços de saúde para retomada da amamentação exclusiva.

Neste estudo, as mulheres com trinta anos e mais apresentaram maior freqüência de introdução precoce de suplementos alimentares. A idade materna pode estar associada ao nível educacional, à paridade e às experiências prévias de aleitamento; por isso, a influência desse fator deve ser analisada com cautela. Também devemos lembrar outros fatores que não foram avaliados, mas podem estar associados à maior freqüência de suplementação precoce: o tabagismo, a inserção no mercado de trabalho, diferentes modos de vida e origens culturais. Uma possibilidade a ser pesquisada é a de essas mulheres terem sido mais expostas à cultura do desmame precoce, que teve seu auge na década de 70 e início da década de 80 .

\section{Considerações Finais}

As limitações do uso de indicadores compostos, como a variável estrato, estão relacionadas ao risco de não escolher adequadamente as variáveis para construção do indicador e o peso que será dado a cada uma delas. Outro problema é saber se a área em que o indicador se baseia é a unidade de planejamento mais apropriada. Em nosso caso, os limites dos setores censitários não consideram a heterogeneidade interna de cada setor; esse fato pode explicar a ausência de diferenças estatisticamente significantes para o aleitamento materno entre os Estratos 1, 2 e 3. Outra limitação a ser considerada é o fato de termos pesquisado apenas os sobreviventes; não sabemos se a inclusão das crianças que faleceram alteraria os dados sobre o aleitamento e sua relação com as demais variáveis. Também devemos levar em conta o alto custo e a complexidade desse tipo de estudo, o que limita sua viabilidade para os serviços de saúde, que exigem avaliações rápidas e de baixo custo, como as que adotam métodos simplificados de amostragem e coleta dos dados.

Este estudo não pode ser utilizado para estabelecer relações causais definitivas, mas pode sugerir fa- 
tores que impedem a amamentação e/ou levam ao desmame precoce, bem como novas direções de pesquisa nas áreas de saúde da criança e avaliação de serviços de saúde.

Este trabalho nos traz também várias perguntas, que levarão a outras pesquisas, e aponta para intervenções possíveis, algumas das quais já em andamento: melhora da qualidade da assistência perinatal, efetivando-se a "Iniciativa Hospital Amigo da Criança"; desenvolvimento de ações de incentivo junto aos grupos identificados como de maior risco crianças de famílias residentes em favelas; que permaneceram em berçário por mais de cinco dias; ou filhos de mães com 30 anos ou mais.

O treinamento dos profissionais de saúde para a promoção do aleitamento materno deve envolver toda a equipe de saúde para obter bons resultados. 19 As atividades de orientação às mães são importantes, mas não suficientes; partilhamos da opinião de Correa ${ }^{20}$ quando afirma que devem ser equacionadas as deficiências e inadequações da assistência à mulher e à criança no que diz respeito à amamentação. A falta de integração dos serviços, a fragmentação da assistência e as dificuldades de treinamento das equipes de saúde têm repercussões não apenas sobre o aleitamento materno, mas também sobre a qualidade de vida da população. Assim, as intervenções dirigidas à melhoria das taxas de aleitamento materno vêm sendo discutidas, no Município de Embu, dentro do contexto da integralidade da atenção à saúde e da humanização dos serviços como garantia do direito à saúde. O presente trabalho pode, portanto, servir de base para uma avaliação futura das ações atualmente em curso, que envolvem a produção e distribuição de material educativo, o treina- mento de profissionais, a reorganização dos serviços de saúde e do sistema de referência e contra-referência com os serviços de maternidade. A atenção à mulher, assim como à família, deve se dar de maneira integral e responder às suas necessidades. As ações de âmbito coletivo, dirigidas a grupos e áreas de maior risco, não se opõem à atenção individual. Na verdade, os dois aspectos se complementam para a promoção, proteção e recuperação da saúde.

A defesa do aleitamento natural, na verdade, deve ser feita através de várias instâncias, entre as quais a discussão do tema nas escolas e o uso dos meios de comunicação social para encorajar o aleitamento e o apoio social à mulher que amamenta, além de propiciar o surgimento de atitudes favoráveis à amamentação entre adolescentes. Esse uso dos meios de comunicação deve ser cauteloso, sem emitir juízos de valor em relação às atitudes maternas, mas com sensibilidade para os valores da comunidade a que se dirige.

Por fim, lembramos que a promoção e proteção ao aleitamento materno deve ser realizada de maneira abrangente e intersetorial, envolvendo todos os níveis de decisão e execução das políticas públicas, assim como a sociedade civil organizada e os meios de comunicação social, na defesa dos direitos da mulher e da criança e de uma melhor qualidade de vida. Amamentar não é um ato isolado, mas parte da vida da mulher e da família; é determinado por uma série de fatores e é também expressão de uma cultura. O sucesso dos esforços de promoção será limitado se não se buscar a valorização da mulher, o respeito à cidadania e a melhora da qualidade de vida, com a satisfação das necessidades da população.

\section{Agradecimentos}

À FAPESP (Fundação de Amparo à Pesquisa do Estado de São Paulo) pelo financiamento da pesquisa, em processo n. 95/3438-7.

\section{Referências}

1. Giugliani ERJ. Amamentação: como e por que promover. J Pediatr [Rio de Janeiro] 1994; 70 : 138-51.

2. Jelliffe DB, Jelliffe EFP. Human milk in the modern world. Oxford: Oxford University Press; 1978.

3. Escuder MML, Venancio SI, Pereira JCR. Estimativa de im-

pacto da amamentação sobre a mortalidade infantil. Rev Saúde Pública 2003; 37: 319-25.

4. Carvalho MR, Bancroft C, Canahuati J, Muxí C. Lactancia materna. In: OPS (Organização Panamericana de la Salud). Acciones de salud maternoinfantil a nivel local: 
segun las metas de la Cumbre Mundial en Favor de la Infancia. Washington (DC); La Organización; 1996. p 249-63.

5. Rea MF, Berquó ES. Impact of the Brazilian national breastfeeding programme on mothers in Greater São Paulo Bull World Health Organ 1990; 68: 365-71.

6. Rea MF. Reflexões sobre a amamentação no Brasil: de como passamos a 10 meses de duração. Cad Saúde Pública 2003; 19 [Supl 1]: 37-45.

7. Pedroso GC, Bracco Neto H, Silva EMK, Puccini RF. Avaliação populacional do aleitamento materno, em "Dia Nacional de Vacinação", no Município de Embu. In: Anais do II Congresso Brasileiro de Pediatria Ambulatorial, V Congresso Brasileiro de Cuidados Primários de Saúde para a Infância e II Congresso Brasileiro de Saúde Escolar; 1996 Abr 30-Maio 3; Fortaleza, Brasil. Fortaleza Sociedade Brasileira de Pediatria; 1996. p. 57. [resumo]

8. WHO (World Health Organization). Indicators for assessing breast-feeding practices: report of an informal meeting. Genebra: The Organization; 1991. (WHO/CDD/ SER/91.14)

9. Puccini RF, Silva NN, Araújo NS, Pedroso GC, Silva EMK. Saúde infantil: condições de vida e utilização de serviços de saúde em área da Região Metropolitana de São Paulo, 1996. Rev Bras Saúde Matern Infant 2002; 2: 143-55.

10. SEADE (Fundação Sistema Estadual de Análise de Dados). Informações dos municípios paulistas: São Paulo em dados). Disponível em: http://www.seade.gov.br/. [2004 jan 31].

11. Frei F. Análise de agrupamentos: estudo metodológico e aplicações em epidemiologia [dissertação mestrado]. São Paulo: Faculdade de Saúde Pública da Universidade de São Paulo; 1998.
12. Pérez-Escamilla R. Patrones de la lactancia natural en América Latina y el Caribe. Bol Ofic Sanit Panam 1993; 115:185-94.

13. Monteiro CA. O panorama da nutrição infantil nos anos 90 . São Paulo: UNICEF; 1996. (Cadernos de Políticas Sociais).

14. Kitoko PM, Rea MF, Venancio SI, Vasconcelos ACCP, Santos EKA, Monteiro CA. Situação do aleitamento materno em duas capitais brasileiras: uma análise comparada. Cad Saúde Pública 2000; 16: 1111-9.

15. Venancio SI, Escuder MML, Kitoko P, Rea MF, Monteiro CA. Freqüência e determinantes do aleitamento materno em municípios do Estado de São Paulo. Rev. Saúde Pública 2002; 36: 313-8.

16. Forman MR. Review of research on the factors associated with choice and duration of infant feeding in less-developed countries. Pediatrics 1984; 74 Suppl: 667-94.

17. Rea MF, Cukier R. Razões de desmame e de introdução da mamadeira: uma abordagem alternativa para seu estudo. Rev Saúde Pública 1988; 22: 184-91.

18. Puccini RF, Pedroso GC, Silva EMK, Araújo NS, Silva NN. Eqüidade na atenção pré-natal e ao parto em área da Região Metropolitana de São Paulo: 1996. Cad Saúde Pública 2003; 19: 35-45.

19. WHO (World Health Organization). Evidence for the ten steps to successful breastfeeding. Geneve: The Organization; 1998. (WHO/CHD/98.9)

20. Correa AMS. Aleitamento materno: estudo sobre o impacto das práticas assistenciais [tese doutorado]. Campinas: Faculdade de Ciências Médicas da Universidade Estadual de Campinas; 1996.

Recebido em 10 dezembro de 2003

Versão final apresentada em 12 de fevereiro de 2004

Aprovado em 23 de fevereiro de 2004 